\title{
Innovative technologies for the smart E-Healthcare system
}

"Oh wow, a dolphin! It looks so real and it's swimming around me." Calming music in the background mingles with the faint sound of ocean water and aquatic animals. Several clown fish slowly move into view and swim in a circle. A cute dolphin beckons, as if to play, and a big whale is seen leisurely swimming in the distance.

Michael, a six-year old boy with burns on his two legs due to recent car accident, is enjoying his deep-sea adventure through smart phone virtual reality (VR), a three-dimensional (3D) computer-generated virtual environment. It was only a week ago when he was living in constant fear and pain from the daily dressing of his burn wounds at the hospital. His physicians needed to prescribe heavy doses of strong intravenous pain medication during these sessions. However, a VR kit was recently recommended as an alternative for Michael's pain management.

For severe, acute, and/or chronic pain, various painkillers, including opioids, are being used. Unfortunately, use of such powerful painkillers can often lead to financial burden and potential drug addiction. Misuse of addictive painkillers leads to the overdosing and death of about 47,000 people a year [1]. More and more physicians are starting to consider VR technologies as an alternative to painkillers. The basic idea behind using VR for pain management is that immersive environment and stimuli can distract patients from their pain and emotional states. In particular, hospitalized and/or bed-bound patients, such as women in labor and patients receiving chemotherapy, dialysis, radiation, or imaging procedures, may have biopsychosocial distress, such as depression, anxiety, and boredom due to their health condition and environment changes. Since 1996, the Harborview Burn Center in Seattle has used VR for burn patients, which has helped distract patients by providing relief and escape from painful treatments [2]. Recent studies from my institute at Cedars-Sinai Medical Center and others explored the use of VR in 100 hospitalized patients and found that those who participated in VR therapy showed a significant reduction in pain scores [3]. This suggests that VR may offer holistic inpatient care by effectively reducing anxiety and pain perception and helping patients relax before, during, or after procedures. Additionally, VR technology is now becoming more userfriendly, cost-effective, interactive, flexible, immersive, and portable. VR is now being considered an effective, safe, and feasible intervention for a variety of disease states, such as obesity, anxiety, eating disorders, post-traumatic stress disorder, cancer, Parkinson's, nicotine/alcohol addiction, cognitive/motor rehabilitation et al. However, the challenge remains on figuring out how to incorporate VR into standard of care procedures in the everyday clinical setting.

One interesting use of VR could be in urology. Medical training using VR and/or AR (augmented reality, an interactive experience of environments where objects in the realworld are "augmented" by computer-generated perceptual information) could improve education and surgical practice, thereby increasing the speed and accuracy of certain procedures. The traditional Halstedian method of "see one, do one, teach one" in medical training could be improved by applying VR/AR-based simulators before performing live surgery. Complicated anatomy can be visualized in a $3 \mathrm{D}$ hologram format, and AR navigation can then facilitate the recognition and localization of anatomic structures. Using a projector beam and a see-through device, such as a smart google, physicians can see the virtual model directly projected on the patient. Furthermore, individual medical records can be reviewed, and images can be portrayed on screens during camera-based AR procedures. VR stimulation training has also been shown to result in superior speed and significant reduction of procedure-related complications. VR/ $\mathrm{AR}$ stimulators can provide a variety of surgical scenarios that can be used during medical training to improve the overall skills required for operations, such as transurethral 
resection of the prostate or prostatectomy. It can record the operative process of trainees in real-time, which can be used for evaluations. Considering how robotic surgical procedures continue to grow rapidly, a computer-derived virtual operative field with a tactile feedback system could be a new reliable standard method in robotic training programs.

A VR/AR simulator can not only be applied for surgeries, but also for personalized treatment of debilitating benign bladder dysfunctions, such as interstitial cystitis (IC)/ painful bladder syndrome. IC is characterized by a need for frequent urination, typically urgent (and often uncontrollable), involuntary episodes, and flares of acute pain without any bacterial or yeast infections [4]. Along with no known etiology, IC has been challenging to urologists because there are no effective pharmacological, surgical, or behavioral treatments available. Current recommendations include exercising specific pelvic floor muscles to reduce severe urinary symptoms. To train unconscious physiological processes, a VR/AR-based biofeedback designed to visualize these exercising muscles could be applied. If patients can see the pelvic floor muscles through a head-mounted display, tablet, or hologram, they can easily understand exactly which muscles they are working and focus their efforts with more specificity; thereby, improving the overall efficacy of the therapy.

Including our own group, many scientific investigators have been developing biomarkers specific to each urological disease using biospecimens from tissues, blood, or other body fluids [5]. It is widely accepted that molecular biomarkers are useful since they can be used for prognoses and treatment response monitoring. Of note, accurate real-time monitoring can be immensely helpful for disease prevention, early diagnosis, and reduction of incidence rates. Biosensors are analytical devices designed to detect biological and physiochemical biomarkers and have been developed into smart health monitoring devices that can wirelessly sync with smartphones [6]. Data collection, storage, and analysis can be done in the connected cloud environment. The key components of potentially successful biosensors in the clinical setting include portability, rapidity, and costeffectiveness. In particular, for efficient application in communication between urologists and patients, more effort should be placed on developing newer generations of highly sensitive molecular diagnostic biosensors with compact and user-friendly platforms.

While blood tests are currently the gold standard for noninvasive, painless, and continuous monitoring of biomarkers, urine is a particularly attractive resource in the urology field. Urine biosensors may provide real- time levels of the patients' urinary metabolite biomarkers, electrolytes, temperature, and $\mathrm{pH}$. There have been studies on developing rapid diagnoses of urinary tract infections (UTIs) in the clinical setting, which would allow for the early identification of UTI and timely antibiotic treatment. UTI is one of the most common infections in women, elders, and infants. Recently, investigators developed a small and disposable UTI biosensor embedded in a diaper. When activated upon exposure to urine, the self-powered sensor can determine the presence of chemical compounds associated with UTI [7]. The data can then be collected via a wireless smartphone app and used for further analysis by urologists and patients, allowing them to discuss the patterns of UTI. For other bladder diseases, such as IC, urinary biosensors may be able to facilitate real-time identification of flares (acute pain) and fluctuation of biomarkers, such as specific metabolites [5]; thereby enabling personalized treatment regimens for patients. Wearable wireless urinary metabolic sensors enable remote tracking of disease biomarkers and bladder health 24/7. They can replace bladder diaries (voiding diaries) and help accelerate interventions regardless of location, time, or device.

Research related to smart health devices is already changing the paradigm of the healthcare industry. Smart healthcare is characterized by eHealth, which is supported by computer-based information and communication technology, and mHealth services, a medical practice supported by mobile devices. Our healthcare system is now rapidly evolving for better patient diagnoses and treatment by establishing the personal digital assistants for electronic record management and smart remote services through biosensor platforms that are wirelessly connected between urologists and patients.

\section{CONFLICTS OF INTEREST}

The authors have nothing to disclose.

\section{ACKNOWLEDGMENTS}

The authors acknowledge support from National Institutes of Health grants (1U01DK103260, 1R01DK100974, U24 DK097154, NIH NCATS UCLA CTSI UL1TR000124), Department of Defense grants (W81XWH-15-1-0415), Centers for Disease Controls and Prevention (1U01DP006079), the US.Egypt Science and Technology Joint Fund by National Academies of Sciences, Engineering, and Medicine (NAS) and The United States Agency for International Development (USAID) (to J.K.). Any opinions, findings, conclusions, or 
recommendations expressed in this article are those of the authors alone, and do not necessarily reflect the views of USAID or NAS. And this research was supported by the International Science and Business Belt Program through the Ministry of Science, ICT and Future Planning (to W.J.K.) (2015-DD-RD-0070).

Wun-Jae Kim¹, Jayoung Kim²

Corresponding Author: Wun-Jae Kim ORCID: https:/orcid.org/0000-0002-8060-8926

${ }^{1}$ Department of Urology, Chungbuk National University College of Medicine, Cheongju, Korea

${ }^{2}$ Departments of Surgery and Biomedical Sciences, Cedars-Sinai Medical Center, Los Angeles, CA, USA

E-mail: wjkim@chungbuk.ac.kr

\section{REFERENCES}

1. Rudman W Jr, Corcoran P, Elliott K. Prescription drug monitoring programs and opioid death rates. JAMA 2017 Nov 28 [Epub]. http://doi.org/10.1001/jama.2017.16300.

2. Escudero LA, Cerutti S, Olsina RA, Salonia JA, Gasquez JA.
Factorial design optimization of experimental variables in the on-line separation/preconcentration of copper in water samples using solid phase extraction and ICP-OES determination. J Hazard Mater 2010;183:218-23.

3. Tashjian VC, Mosadeghi S, Howard AR, Lopez M, Dupuy T, Reid M, et al. Virtual reality for management of pain in hospitalized patients: results of a controlled trial. JMIR Ment Health 2017 Mar 29 [Epub]. http://doi.org/10.2196/mental.7387.

4. McLennan MT. Interstitial cystitis: epidemiology, pathophysiology, and clinical presentation. Obstet Gynecol Clin North Am 2014;41:385-95.

5. Wen H, Lee T, You S, Park SH, Song H, Eilber KS, et al. Urinary metabolite profiling combined with computational analysis predicts interstitial cystitis-associated candidate biomarkers. J Proteome Res 2015;14:541-8.

6. Jayanthi VSPKSA, Das AB, Saxena U. Recent advances in biosensor development for the detection of cancer biomarkers. Biosens Bioelectron 2017;91:15-23.

7. Yu W, Seo W, Tan T, Jung B, Ziaie B. A diaper-embedded disposable nitrite sensor with integrated on-board urine-activated battery for UTI screening. Conf Proc IEEE Eng Med Biol Soc 2016;2016:303-6. 